\title{
Identifying the progenitor set of present-day early-type galaxies: a view from the standard model
}

\author{
S. Kaviraj ${ }^{1,2}$, J. E. G. Devriendt ${ }^{2,3}$, I. Ferreras ${ }^{1}$, S. K. Yi ${ }^{4}$, and J. Silk ${ }^{2}$ \\ 1 Mullard Space Science Laboratory, Holmbury St. Mary, Dorking, Surrey RH5 6NT, UK \\ e-mail: skaviraj@astro.ox.ac.uk \\ 2 Department of Physics, University of Oxford, Keble Road, Oxford OX1 3RH, UK \\ 3 Observatoire Astronomique de Lyon, 9 Avenue Charles André, 69561 Saint-Genis Laval Cedex, France \\ ${ }^{4}$ Center for Space Astrophysics, Yonsei University, 134 Shinchon, Seoul 120-749, Korea
}

Received 30 June 2008 / Accepted 9 June 2009

\begin{abstract}
We present a comprehensive theoretical study, using a semi-analytical model within the standard LCDM framework, of the photometric properties of the progenitors of present-day early-type galaxies in the redshift range $0<z<1$. We explore progenitors of all morphologies and study their characteristics as a function of the luminosity and local environment of the early-type remnant at $z=0$. In agreement with previous studies, we find that, while larger early-types are generally assembled later, their luminosity-weighted stellar ages are typically older. In dense cluster-like environments, $\sim 70$ percent of early-type systems are "in place" by $z=1$ and evolve without interactions thereafter, while in the field the corresponding value is $\sim 30$ percent. Averaging across all environments at $z \sim 1$, less than 50 percent of the stellar mass which ends up in early-types today is actually in early-type progenitors at this redshift, in agreement with recent observational work. The corresponding value is $\sim 65$ percent in clusters, due to faster morphological evolution in such dense environments. We develop probabilistic prescriptions which provide a means of including spiral (i.e. non early-type) progenitors at intermediate and high redshifts, based on their luminosity and optical colours. For example, we find that, at intermediate redshifts $(z \sim 0.5)$, large $\left(M_{V}<-21.5\right)$, red $(B-V>0.7)$ spirals have $\sim 75-95$ percent chance of being an early-type progenitor, while the corresponding probability for large blue spirals $\left(M_{B}<-21.5, B-V<0.7\right)$ is $\sim 50-75$ percent. The prescriptions developed here can be used to address, from the perspective of the standard model, the issue of "progenitor bias", whereby the exclusion of late-type progenitors in observational studies can lead to inaccurate conclusions regarding the evolution of the early-type population over cosmic time. Finally, we explore the correspondence between the true "progenitor set" of the present-day early-type population - defined as the set of all galaxies that are progenitors of present-day early-types regardless of their morphologies - and the frequently used "red-sequence", defined as the set of galaxies within the part of the colour-magnitude space which is dominated by early-type objects. We find that, while more massive members $\left(M_{V} \leq-21\right)$ of the "red sequence" trace the progenitor set reasonably well, the relationship breaks down at fainter luminosities $\left(M_{V} \geq-21\right)$. Thus, while the results of recent observational studies which exploit the red sequence are valid (since they are largely restricted to massive galaxies), more care should be taken when deeper observations (which will probe fainter luminosities) become available in the future.
\end{abstract}

Key words. galaxies: elliptical and lenticular, $\mathrm{cD}$ - galaxies: evolution - Galaxy: formation - galaxies: fundamental parameters

\section{Introduction}

As "end points" of galaxy merger sequences, early-type galaxies carry important signatures of mass assembly and star formation in the Universe. Deducing their star formation histories (SFHs) therefore contains the key to understanding not only the evolution of these galaxies but the evolutionary patterns of galaxies as a whole. Our view of early-type galaxy formation has developed over the years, away from the classical "monolithic collapse" hypothesis (e.g. Larson 1975; Chiosi \& Carraro 2002) and towards the hierarchical assembly of these objects through mergers and accretion of smaller galaxies over time, in the framework of the currently popular LCDM paradigm of galaxy formation (e.g. Kauffmann et al. 1993, 1996; Baugh et al. 1996; Kauffmann \& Charlot 1998; Somerville \& Primack 1999; Cole et al. 2000; Hatton et al. 2003; De Lucia et al. 2006; Bower et al. 2006, and references therein).

A significant body of observational work in the past has traced the assembly histories of early-type galaxies by studying only early-type populations at high redshift (e.g. Gladders et al. 1998; Stanford et al. 1998; Barrientos \& Lilly 2003;
Ferreras et al. 2005). However, a fundamental feature of earlytype formation in the standard LCDM model is that stellar mass that eventually ends up in present-day early-type galaxies is not entirely contained in early-type systems at high redshift. Although, early-types at any redshift are almost guaranteed progenitors of their counterparts at present-day, looking only at early-types at high redshift introduces a "progenitor bias", which becomes increasingly more severe at larger look-back times, as the fraction of early-type galaxies becomes progressively smaller and late-type systems begin to dominate the progenitor population (e.g. Franx \& van Dokkum 1996; van Dokkum et al. 2000; van Dokkum \& Franx 2001; Kaviraj et al. 2005).

In the current era of large scale surveys, e.g. SDSS (Adelman-McCarthy et al. 2006), COMBO-17 (Wolf et al. 2004), MUSYC (Gawiser et al. 2006), GEMS (Rix et al. 2004), unprecedented amounts of data spanning a large range in redshift (typically $0<z<1$ ) and environment are becoming available, allowing us to study statistically significant numbers of galaxies at various stages of evolution. A quantitative study of earlytype progenitors within the standard model is therefore desirable 
to: (a) understand the difference in the way early-types are assembled as a function of their luminosity and environment and; (b) to gauge the role of non-early-type progenitors in early-type evolution, especially for studies that focus at high redshift.

A central theme of this work is to use a model which accurately reproduces the photometric properties of early-type galaxies and their observed evolution over a large range in redshift to study, in detail, the properties of the progenitors of early-type galaxies at present day. The process of mapping the progenitor population and its evolution with redshift also provides a realistic picture of progenitor bias within the framework of the standard model.

While van Dokkum \& Franx (2001) have developed and studied the issue of progenitor bias, their study employed phenomenological SFHs, with the simple (and perhaps unrealistic) assumption that morphological transformations occur abruptly $\sim 1.5$ Gyr after the cessation of star formation in a particular galaxy. Their work was an extension of previous ideas (e.g Bower et al. 1998; Shioya \& Bekki 1998) that took into account the potential for elliptical galaxies to have complex star formation histories, but did not explore the effect of morphological transformations on the evolution of the early-type population at high redshift.

While some recent observational studies (e.g. Holden et al. 2005; van de Ven et al. 2003; van Dokkum \& van der Marel 2007) have used the results of van Dokkum \& Franx (2001) to take the effects of progenitor bias into account, other studies (Blakeslee et al. 2003) have used the entire galaxy population, without reference to morphology, in an attempt to reduce the bias in their conclusions that would occur if only elliptical galaxies were used in the analysis.

Our study refines and extends the results of van Dokkum \& Franx (2001) by studying early-type progenitors within a realistic and well-calibrated semi-analytical framework, in which mass assembly and morphological transformations can be followed more accurately in the context of the LCDM paradigm. While the van Dokkum \& Franx (2001) study does attempt to alleviate the effects of progenitor bias, its simplicity, especially in the prescription used for morphological transformations, makes a more realistic treatment of this issue, in the framework of the standard model, very desirable. Using the entire galaxy population without reference to morphology is also not ideal because that implies that all galaxies at high redshift are potential progenitors of present-day early-type galaxies. While this approximation may be reasonably robust for the most massive early-type galaxies in the densest regions of the Universe, our study shows that this is generally not applicable.

In this paper we study the evolution of the "progenitor set" of early-type galaxies, defined as the set of all galaxies at a given redshift that are, regardless of morphology, the progenitors of an early-type at $z=0$. Note that "early-types" are defined as galaxies that are elliptical or lenticular. See Sect. 2 for a description of how galaxy morphologies are defined in the model. We explore the evolution of the progenitor set with redshift, as a function of the luminosity and environment of the early-type remnant which is left at present-day. We pay particular attention to spiral progenitors in the model, since these might have been excluded from some studies of early-type evolution in the past, by virtue of their morphology, even though they form an important part of the progenitor set. By comparing the properties (optical colours and luminosities) of spiral progenitors to the general spiral population, we provide a means of correcting for progenitor bias, which is consistent with the properties of the standard model, by including specific parts of the spiral population at high redshift into the study of early-type evolution.

The plan of this paper is as follows. In Sect. 2, we describe the salient features of the model used in this study. In Sect. 3, we quantify the morphologies of the galaxies that make up the progenitor set. We map the properties of elliptical, S0 and spiral progenitors as a function of redshift and explore differences between progenitors as a function of the mass and environment of the elliptical remnant at present day. In Sect. 4 we focus exclusively on spiral progenitors and compare their photometric properties to the general spiral population, to derive probabilistic prescriptions for including spiral progenitors in early-type studies. Section 5 traces the contribution of galaxies in dense regions at high redshift to cluster early-types at present-day. Finally, in Sect. 6 we explore the correspondence between the true progenitor set of present-day early-type galaxies and the "red-sequence", which is sometimes used as a proxy for the progenitor set in observational studies (e.g. Bell et al. 2004; Faber et al. 2007).

Note that throughout this study we provide rest-frame magnitudes for all model galaxies. Unless otherwise noted, the filters used are in the standard Johnson system. Note that colour figures are available in the online version of this paper.

\section{The model}

The semi-analytical model used in this study is GALICS, which combines large-scale cosmological $N$-body simulations with analytical recipes for the evolution of baryons within dark matter haloes (Hatton et al. 2003). GALICS makes predictions for the overall statistical properties of galaxy populations, with an emphasis on their panchromatic spectral energy distributions across a wide wavelength range ( $U V$ to infrared/submillimetre). In this section we describe the salient features of the model, including the implementation of the cosmological simulation and details of the semi-analytics that determine the growth of bulges. We also highlight the successes of GALICS in terms of reproducing the photometric properties of early-type galaxies in the $U V$-optical spectral ranges across a wide redshift range.

Before we begin our analysis we briefly describe the definition of galaxy morphology in the model used in this study. Galaxy morphology in the model is determined by the ratio of the $B$-band luminosities of the disk and bulge components which correlates well with Hubble type (Simien \& de Vaucouleurs 1986). A morphology index is defined as

$I=\exp \left(\frac{-L_{B}}{L_{D}}\right)$

such that a pure disk has $I=1$ and a pure bulge has $I=0$. Following Baugh et al. (1996), ellipticals have $I<0.219$, S0s have $0.219<I<0.507$ and spirals have $I>0.507$.

\subsection{Dark matter simulation}

The dark matter "backbone" used in GALICS has been generated by simulating a LCDM model in a cube with a comoving size of $100 \mathrm{~h}^{-1}$ Mpc with parameters $\Omega_{\mathrm{m}}=0.33$, $\Omega_{\Lambda}=0.667$ and $\sigma_{8}=0.88$. The amplitude of the power spectrum is computed using the present-day abundance of rich clusters (Eke et al. 1996) and initial conditions are extracted using the GRAFIC code (Bertschinger 1985). The simulation contains $256^{3}$ dark matter particles. The mass of each dark matter particle is $\sim 8 \times 10^{9} M_{\odot}$ and the spatial resolution achieved is $\sim 29.3 \mathrm{kpc}$. 


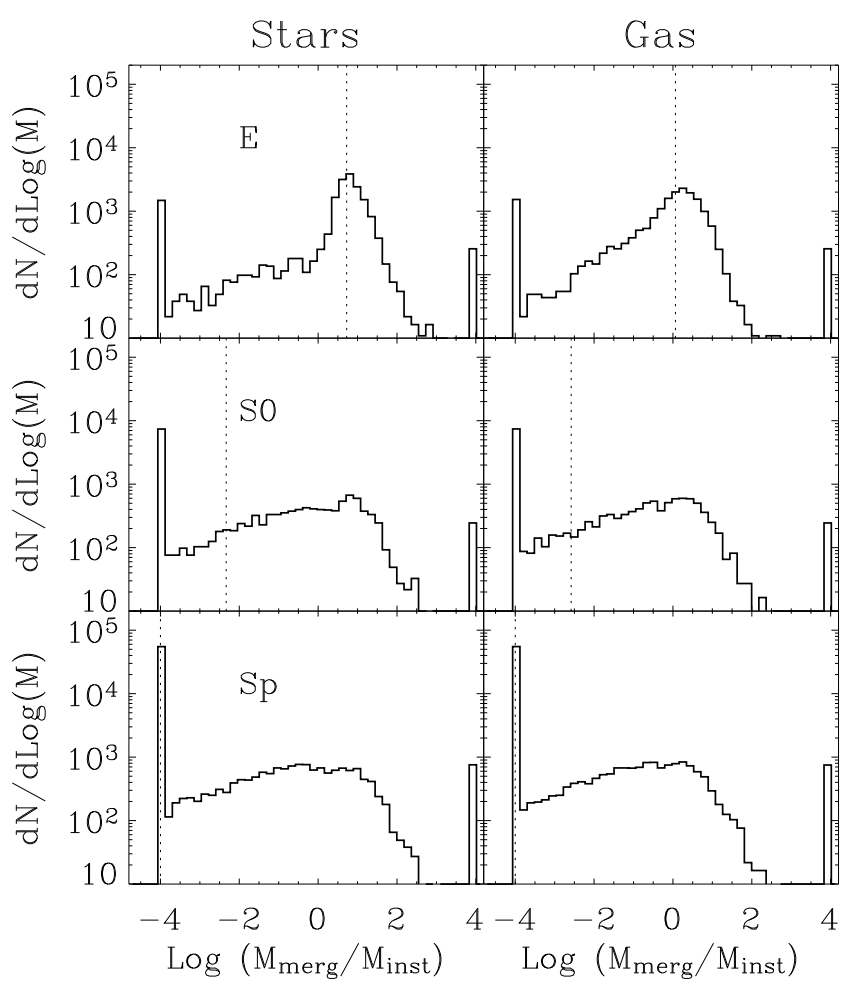

Fig. 1. The ratio between stellar and gas masses transferred from the disk to the bulge during mergers $\left(M_{\text {merg }}\right)$ and through disk instabilities $\left(M_{\text {inst }}\right)$. A galaxy with a value of 1 for this ratio has acquired half its bulge mass during mergers and the other half through disk instabilities. This plot is restricted to model galaxies brighter than $M(B)=-18.9$. Note that the (artificial) peaks at 4 and -4 are for ratios $M_{\text {merg }} / M_{\text {inst }}=\infty$ and 0 respectively. The vertical dashed lines represent median values.

The minimum mass of galaxies (due to the minimum number of particles used in the friend-of-friends group finder and the baryon fraction used) is $\sim 2 \times 10^{10} M_{\odot}$.

\subsection{The growth of bulges in the semi-analytical model}

\subsubsection{Mergers vs. disk instabilities}

GALICS includes two processes which lead to the formation and growth of spheroidal components in galaxies: mergers (both major and minor) and gravitational disk instabilities ${ }^{1}$. While mergers directly reveal the hierarchical nature of galaxy formation, disk instabilities can result in the formation of bulges in a "monolithic" manner (i.e. in objects classified as isolated at our resolution limit). The relative contribution of each of these two processes in the build-up of bright, local galaxy bulges thus yields a natural estimate of how hierarchical galaxy formation truly is.

For each of our galaxies we measure the stellar and gas masses transferred from the disk to the bulge during mergers $\left(M_{\text {merg }}\right)$ and/or disk instabilities $\left(M_{\text {inst }}\right)$. In Fig. 1, we show the relative contributions of mergers (both major and minor) and disk instabilities to the mass build-up of the spheroidal components of local galaxies brighter than $M(B)=-18.9$. This is shown as the distribution of the ratio $M_{\text {merg }} / M_{\text {inst }}$, computed for each model galaxy. A galaxy with a value of 1 for this ratio

\footnotetext{
1 A disk is considered to be stable if $V_{c}<0.7 V_{\text {tot }}$, where $V_{\mathrm{c}}$ is the circular velocity of the disk and $V_{\text {tot }}$ is the rotational velocity of the disk-bulge-halo system (e.g. van den Bosch 1998).
}

has acquired half its bulge mass during mergers and the other half through disk instabilities. Note that we only consider galaxies with a bulge component. Pure disk galaxies (i.e. those that have never been unstable or undergone a merger) represent only $\sim 1.4$ percent of the bright population.

Several important conclusions can be drawn from this figure. First, it is clear that the majority of elliptical galaxies develop their bulges during mergers rather than disk instabilities. This result may seem natural, as GALICS is an implementation of the hierarchical galaxy formation scenario. However, the main point here is that, for the first time, we do indeed compare the relative contribution of mergers to other processes in the formation of spheroids. The fact that model elliptical galaxies develop their bulges mainly through mergers rather than through disk instabilities is an output of our model - we do not simply assume that elliptical galaxies only form during mergers but instead allow the different physical processes we believe are relevant to the formation process to compete. In that sense, one can consider the results we obtain as a demonstration that the formation of ellipticals is indeed hierarchical.

Second, elliptical and lenticular galaxy formation appears to be somewhat different, since GALICS predicts that most lenticular galaxies develop their bulges through disk instabilities. Changing the modelling of disk instabilities would affect the plots in a similar way for ellipticals and lenticulars, leaving the difference in their respective behaviours unchanged. The fact that the model lenticulars acquire their morphology through disk instabilities is a result which is common in numerical simulations of galactic dynamics (Combes et al. 2000) and gives us some confidence that our simple physical modelling broadly captures the mechanism.

Finally, the lower panels of Fig. 1 indicate that spirals form their bulge components mainly through disk instabilities. The global picture for morphogenesis that we obtain from GALICS is satisfactory as it compares well to more detailed numerical simulations. Moreover, we show that whilst an "isolated object" approximation can be justified to describe spiral galaxies (and some lenticulars), it cannot be used to properly model the formation of the vast majority of ellipticals in the framework of CDM structure formation.

\subsubsection{A note on the treatment of disk instabilities}

The disk instability model employed is similar to that of van den Bosch (1998). Rotational equilibrium is enforced at the disk half mass radius - in other words, the circular velocity $V_{\text {tot }}$ arising from the presence of total mass of gas, stars and dark matter enclosed in the half mass radius sphere is calculated. It is then compared to the circular velocity $V_{\mathrm{c}}$ of the disk alone, and if this latter is smaller than a critical value (obtained by mass weighting the value of 0.7 for a pure gas disk and 0.52 for a pure stellar disk), the minimum amount of mass of gas and stars required to re-establish stability is transferred to the disk.

\subsubsection{Post-merger morphology}

In the literature mergers are typically modelled by taking the ratio of the progenitors, adding the stars of the lighter galaxy to the disk of the heavier one if the mass ratio is less than $f_{\text {bulge }} \sim 0.3$ or destroy the disk and form a bulge if the ratio is higher (e.g. Walker et al. 1996; Cole et al. 2000; Somerville \& Primack 1999; 
Table 1. Free parameters that affect the evolution of the baryonic Universe, see Sect. 2.3 for more details.

\begin{tabular}{cccc}
\hline \hline Parameter & Description & Fiducial value & Source of constraints \\
\hline$\beta$ & Inverse of star formation efficiency & 50 & Kennicutt (1998) \\
$\epsilon$ & Inverse of mass loading for feedback & 0.1 & Martin et al. (2002) \\
$\chi$ & Galaxy merger power law & 3.333 & Numerical simulations e.g. Walker et al. (1996) \\
\hline$\Omega_{B}$ & Baryon fraction & $0.02 \mathrm{~h}^{-2}$ & ${ }^{2} \mathrm{H}$ abundance in QSO absorption lines (Tytler et al. 1996) \\
$\psi$ & S-S merging normalisation & 0.017 & Makino \& Hut (1997) \\
$S$ & Recycling efficiency & 0.3 & Set to fiducial value in Hatton et al. (2003) \\
$\kappa$ & Ratio of burst-to-bulge radius & 0.1 & Set to fiducial value in Hatton et al. (2003) \\
Dust & ISM Extinction and emission due to dust & - & Guiderdoni et al. (1987), Desert et al. (1990) \\
IMF & Initial mass function & - & Kennicutt (1983) \\
\hline
\end{tabular}

Kauffmann et al. 1999) $)^{2}$. Since a galactic disk can be disrupted by an encounter even when the interloper is less massive than the disk itself, this simple prescription reproduces this behaviour. However, the sharp cut-off at $f_{\text {bulge }} \sim 0.3$ between totally disrupting or not disrupting the morphology of the galaxy seems somewhat unrealistic.

The model implemented in GALICS is constructed in terms of a smooth function $(X)$, which models the fraction of disk material remaining in the disk after the merger as a function of the mass ratio of the two progenitors. The previous studies (described above) effectively use a step function, in the sense that the material kept in the disk is either 0 or 1 depending on whether the mass ratio is greater or lesser than 0.3 respectively. In GALICS this smooth function is defined as:

$X(R)=\left[1+\left(\frac{\chi-1}{R-1}\right)^{\chi}\right]^{-1}$,

where $R$ represents the mass ratio of the heavier to the lighter progenitor and $\chi$ is the critical value of that ratio i.e. the value that $X=0.5$. Since step functions with $f_{\text {bulge }} \sim 0.3$ have been found to give good results in the past, the fiducial value adopted in GALICS is $\chi=1 / 0.3$. See Fig. 3 in Hatton et al. (2003) for a visualisation of $X(R)$ for this fiducial value of $\chi$. We refer readers to Sect. 5 in Hatton et al. (2003) for details of prescriptions related to merging employed by GALICS.

Galaxies are modelled with three components: the disk, the bulge and the burst. The burst has the same geometry (and therefore star formation law) as the bulge but its scale radius is $10 \%$ of that of the bulge. During a merger, a fraction of $X$ of both gas and stars originally in the disk remain in the disk while the rest are transferred to the burst. Stars formed/existing in the burst are rapidly transferred to the bulge. As the central burst forms stars (typically over short timescales than the "quiescent" mode due to its smaller scale radius), the new stellar component gets transferred to the bulge. As it subsequently runs out of the gas deposited by the merger, the burst eventually disappears. We refer readers to Sect. 5 of Hatton et al. (2003) for further details. Note that, for higher values of $R$ (i.e. mergers with a high mass ratio) almost all disk material remains in the disk, while equal mass mergers result in rapid bulge formation, mimicking the prescriptions employed in the literature.

\subsection{A discussion of free parameters in the model}

Inevitably, any study that exploits a semi-analytical model such as the one presented here, relies on simplified, albeit

\footnotetext{
2 In keeping with the literature, we define a merger as "major" when the mass ratio between merging objects ranges between one and a third, and as "minor" when it is smaller.
}

well-calibrated recipes to describe galaxy evolution. These semianalytic recipes are driven by free parameters which determine the evolution of the baryonic Universe. While the parameters that drive the baryonic evolution in the fiducial GALICS model are discussed in Sect. 7 of Hatton et al. (2003), we briefly revisit them here and discuss their potential impact on the analysis that follows in the paper. In Table 1 we list the free parameters in the model and indicate how their values are constrained in this study. The first section of the table indicates parameters that have the greatest impact on the evolution of the galaxy population and the bottom section indicates parameters that play a minor or negligible role.

While the model is driven by several free parameters, those that have the greatest impact on the spectro-photometric properties of the galaxy population are: (a) the star formation efficiency; (b) the efficiency of mass loading for supernova feedback; and (c) the merging law that determines morphological transformations during galaxy interactions. We explore each of these in turn and briefly discuss the sensitivity of the model to these parameters.

Star formation efficiency: the star formation efficiency (SFE) determines the rate at which cold gas is converted into stars. For example, decreasing the SFE results in less cold gas being converted into stars, moves the peak of the star formation rate closer to present day and affects both the shape and normalisation of the $z=0$ galaxy luminosity function. In our fiducial model, the inverse of the $\operatorname{SFE}(\beta)$ is set equal to 50 (i.e. the SFE is $\sim 2 \%$ ), following Kennicutt (1998, see their Eq. (7)), who combined $\mathrm{H} \alpha, \mathrm{HI}, \mathrm{CO}$ and far-infrared measurements in spiral and infrared-selected starburst galaxies to provide a parametrisation of the global star formation rate in the local Universe. Figure 6 in their study indicates that this parametrisation is accurate over a wide gas density range, from gas-poor spiral disks to the cores of the most luminous starburst galaxies, implying that the value for this parameter that is used in the model is reasonably well-constrained. Furthermore, the present-day $B$ and $K$-band galaxy luminosity functions predicted by GALICS are very consistent with those observed by the $2 \mathrm{dF}$ survey (Cross et al. 2001), indicating that the effect of the adopted value $(\beta=50)$ is consistent with the observed distribution of galaxy luminosities in the real Universe.

Mass loading for supernova feedback: for a given stellar mass formed, a certain fraction is contained in massive, fast-evolving stars that become supernovae (SN), injecting kinetic energy into the interstellar medium. The efficiency of this SN driven "wind" depends on both the porosity of the ISM (see Silk 2001) and the "mass loading factor", which describes the entrainment of interstellar gas by the wind 
(see e.g. Silk 2001, 2003). For example, increasing the mass loading efficiency will produce more feedback, heat more cold gas and reduce the amount of fuel available for star formation. This will, in turn, affect the galaxy luminosity function at present-day. Following Martin et al. (2002), we assume that the mass loading factor is $\sim 10$, which results in the outflow rate of a starburst to be of the order of the star formation rate (see Sect. 4.2 in Hatton et al. 2003 for more details). Thus the inverse of the mass loading factor $(\epsilon)$ is set to 0.1 . As mentioned before, the reproduction of the galaxy luminosity functions in the $B$ and $K$ bands - which relies in part on $\epsilon-$ are well reproduced by the fiducial GALICS model.

Merger law: a third parameter that has a significant impact on galaxy evolution is the merger law that determines postmerger morphology. This law determines the morphological mix of the Universe at $z=0$ and therefore has a direct impact on the early-type progenitor set in the model. The prescription used to determine post-merger morphology has already been described in detail above in Sect. 2.2.3. Hatton et al. (2003) indicates that the predicted morphological mix in GALICS is (within counting errors) consistent with that seen in the real Universe. Note, however, that the comparisons presented in Sect. 8.6 of Hatton et al. (2003) use reasonably small surveys (e.g. the Stromlo-APM redshift survey; Loveday et al. 1996) compared to the scale of modern surveys such as the SDSS. Future papers will present more robust comparisons between the morphological mix predicted by GALICS and that observed in the SDSS e.g. through comparison of the GALICS predictions to visually-inspected morphologies of the entire SDSS DR6 measured by the "Galaxy Zoo" project (Lintott et al. 2008).

Minor parameters: we complete our description of the free parameters by briefly discussing minor parameters that do not produce a measurable impact on the analysis. The normalization for the satellite-satellite merging law $(\psi)$ is constrained using Makino \& Hut (1997). Satellite-satellite merging is much rarer than dynamical friction merging (i.e. merging with centrals in a halo) and thus this parameter plays a negligible role in the analysis.

The recycling efficiency $(\varsigma)$ describes the fraction of gas originally expelled from the halo by feedback which is reaccreted over time as the dark matter (DM) halo grows in mass. We note that Somerville \& Primack (1999) set this parameter to 0 (i.e. any gas expelled is lost from the system altogether) while Cole et al. (2000) set it to 1. Hatton et al. (2003) use a fiducial value of $30 \%$, to represent an intermediate behaviour compared to the two extremes described above, which is plausibly closer to the truth. We note, however, that the effect of this parameter is very weak because at the mass resolution of the model, very little gas is expelled from DM halos. The re-accreted gas is always significantly less than the amount of "pristine" gas accreted or the amount of gas that is ejected out of the galaxy and into the halo in the first place.

In GALICS star formation driven by disk instabilities and mergers takes place in a central "burst" region, which is modelled as having the same morphology as the bulge but with a fraction of its radius (see Sect. 4 in Hatton et al. 2003 for a more details). The "burst to bulge" radius $(\kappa)-$ set to a fiducial value of 0.1 - dictates the size of the burst region in model galaxies (i.e. an order of magnitude smaller than the radius of the bulge). Reducing $\kappa$ would make the burst regions smaller, shortening the star formation timescales (and vice-versa). However, typical star formation timescales (a few tens of Myr at most) in the burst regions are negligible compared to the timescales over which galaxy properties are being "viewed" in the model (several Gyr). Hence, changing this parameter does not affect the observed colours of the galaxy population predicted by the model and thus leaves our conclusions unchanged.

The dust recipe used in the model is calibrated using the Milky Way, the Large Magellanic Cloud and Small Magellanic Cloud and a few local spirals for which the extinction curve, gas content and metallicity have been measured (see Guiderdoni \& Rocca-Volmerange 1987, for details). While a key assumption is that the dust properties of galaxies are invariant with redshift, tests of this assumption requires data at high redshift which are not yet available, leaving us very little room to further calibrate our dust recipe. Finally, the fiducial GALICS model uses the Kennicutt (1983) Initial Mass Function (IMF). We note that the dispersion in the predicted properties of the early-type population in the local Universe does not vary significantly with other similar IMFs such as Salpeter (1955) - see Table 1 in Kaviraj et al. (2005).

Notwithstanding the large set of free parameters inherent to any semi-analytical analysis, we note that the fiducial values of the parameters in the model are calibrated either through observational data (e.g. the SF efficiency) or through numerical simulations (e.g. the merger law). Most importantly, the full suite of calibrated parameters that drives the model predictions reproduces a fundamental set of spectro-photometric properties of the observed galaxy population in the Universe. GALICS produces good agreement to the galaxy luminosity functions observed by the $2 \mathrm{dF}$ survey in the $B$ and $K$ bands (Cross et al. 2001). The $(B-V)$ colours of spiral galaxies closely follow the observed data of Buta et al. (1994), both in terms of average values and scatter. Satisfactory fits to the Faber-Jackson relation for early-types (and the Tully-Fisher relation for disks) are obtained and predictions show good agreement with the early-type Fundamental Plane, with the model predicting the observed morphological mix of the local Universe with a reasonable degree of accuracy. As we describe in detail in the next section, GALICS has been specifically tested against a wide variety of multi-wavelength (UV-optical) early-type galaxy properties across a wide range in redshift $(0<z<1.5)$, making it the ideal tool to map the early-type progenitor set over the redshift range $0<z<1$.

We now turn briefly to the impact of the free parameters on our subsequent analysis and explore the potential uncertainty in our results, given the freedom that we may have in the values of the parameters in the model. Recalling that the primary aim of the paper is to map the photometric properties of the early-type progenitor set, we note that the parameters that potentially affect the analysis the most are: (a) those that are responsible for the morphological mix of the Universe (since this determines the composition of the progenitor set at any given time); and (b) those that strongly affect galaxy luminosities/colours (since the probabilistic prescriptions designed to include late-type progenitors are given as a function of luminosities and colours).

As described above the key parameter that drives morphology is the merger law, in particular the transition mass ratio $(\sim 1: 3)$ at which morphological transformations takes place (i.e. disks are disrupted and bulges form). However, the merger recipe is constrained reasonably robustly through numerical 
simulations and the galaxy population predicted by the model is consistent with the morphological mix of observed Universe, indicating that the composition of the progenitor set is predicted with an acceptable level of accuracy across our target redshift range.

In a similar vein, galaxy luminosities/colours are driven by a suite of parameters including the SF efficiency, SN feedback, IMF and dust recipes. This set of parameters is constrained through comparison of the fiducial model to the properties of observed galaxies. While we have, in principle, freedom to change these parameters, calibrations to multiple observational facts in the real Universe, such as the ones described in the previous section, severely reduce this freedom in practice. In other words, if individual parameters are altered arbitrarily then the reproduction of the galaxy properties in the model would fail. In this sense, the analysis presented in the paper is stable, in that they are driven by values of the free parameters that are based either on observations/hydrodynamical simulations and which reproduce the galaxy properties in the present-day Universe with a reasonable degree of accuracy.

\subsection{Reproduction of the early-type galaxy population}

Since our aim is to map the photometric properties of earlytype progenitors, it is important that the model reproduces the multi-wavelength properties of the early-type population and their evolution with redshift. A well-calibrated model is clearly needed for the progenitor predictions to be reliable. Note that in the redshift interval $(z<1)$ and for the wavelength range ( $B$ and $V$ band) studied in this paper, the population synthesis models employed by the fiducial GALICS model - STARDUST (Devriendt et al. 1999) - provide virtually identical results to other commonly used models (Yi 2003; Bruzual \& Charlot 2003; Maraston 2005).

In addition to the reproduction of the general galaxy population, the fiducial GALICS model has been specifically tested in the context of early-type galaxies, across virtually the entire redshift range over which early-types have been observed $(0<z<1.5)$. GALICS accurately reproduces the optical colour magnitude relations (CMRs) of the early-type population (in dense environments) and their evolution from $z=0$ to $z \sim 1.23$ (Kaviraj et al. 2005). The predicted evolution of both the gradient and the scatter in the optical CMRs are consistent within errors with various observational studies which use a variety of optical colours (see Fig. 8 in Kaviraj et al. 2005). More massive ellipticals are predicted to be older (although they assemble more recently) and more metal-rich than their less massive counterparts (see Fig. 3 in Kaviraj et al. 2005). The star formation histories of elliptical galaxies are shown to be quasi-monolithic, which enables the model to maintain the correct gradient and scatter over the entire range in redshift $(0<z<1.27)$ at which observational early-type studies have been conducted (see Fig. 5 in Kaviraj et al. 2005). Note that the decoupling of the assembly history of elliptical galaxies from their star formation history described above has also been found in other semi-analytical work (e.g. Kauffmann et al. 1996; Baugh et al. 1996; De Lucia et al. 2006), although they do not study the evolution of the optical colours with redshift. Finally, GALICS is the only semi-analytic model to have been tested against the new generation of $U V$ photometric data, shortward of $3000 \AA$, made available by the recent GALEX mission (Martin et al. 2005). Kaviraj et al. (2007) find excellent quantitative agreement between the predictions of this model and the observed $U V$-optical photometry of $\sim 2100$ early-type galaxies in the nearby $(0<z<0.11)$ Universe that have been imaged by both GALEX and the SDSS DR3.

Given its good reproduction of early-type photometry across a wide wavelength range and its successful prediction of earlytype colour evolution to high redshifts $(z \sim 1.23)$, GALICS is a useful and well-calibrated tool with which to follow the progenitor set of early-type galaxies and the evolution of that progenitor set in the redshift range $0<z<1$. We note that the emphasis in this study is not to focus on the properties (e.g. star formation and assembly histories) of early-type remnants at $z=0$ (which is the major thrust of previous semi-analytical studies of elliptical galaxies), but to focus on the properties of the progenitor galaxies over the redshift range covered by the bulk of the recent and forthcoming observational surveys $(0<z<1)$.

\section{Dissecting the progenitor set: morphologies of progenitors and epochs of last mergers}

Early-type galaxies have an assortment of star formation histories (SFHs). Central to this study is the epoch at which the last merger, that finally creates the early-type remnant, takes place. After this event, the early-type remnant evolves to present day without further interactions with other galaxies. In the discussion that follows, we refer to the look-back time to this "last-merger" as the dynamical age of a galaxy. Note that the last mergers typically have mass ratios of 1:5 or higher.

As our subsequent analysis of the progenitor population involves environments of model galaxies, an explanation of the definition of this quantity is necessary. Galaxy environments in the model are driven by the mass of the dark matter (DM) halo in which they are embedded. At $z=0$, DM halo masses greater than $\sim 10^{14} M_{\odot}$ correspond to "cluster" environments, while halo masses between $\sim 10^{13} M_{\odot}$ and $\sim 10^{14} M_{\odot}$ correspond to "groups". All other halo masses correspond to the "field". At higher redshifts these definitions do not strictly hold since the DM halo population is evolving - for example, the largest haloes at $z=1$ are likely to be roughly half their size at present day (e.g. van den Bosch 2002). We take this mass accretion history into account when specifying the environments of galaxies at high redshift. The mass accretion history is taken from van den Bosch (2002, see their Fig. 5).

The number of early-types galaxies in the simulation, based on the definitions presented above, is 5418. 279 early-types are in clusters $(\sim 5 \%), 1081(\sim 20 \%)$ are in groups and $4058(\sim 75 \%)$ are in the field. At $z=0$ there are 8 clusters identified in the GALICS simulation box.

Figure 2 indicates the last merger redshifts of the sample of early-types in the model, split by the environment of the remnant at $z=0$. Recall that after the last merger, the early-type remnant evolves to present day without further interactions with other galaxies. The inset in Fig. 2 shows histograms of the last merger redshifts, again split by environment. As expected, we find that, in all environments, larger early-types are assembled later although their stars are generally older (Kaviraj et al. 2005). This result is consistent with the findings of De Lucia et al. (2006), although we note that they did not consider early-types in different environments separately.

Cluster galaxies (at least those brighter than $L_{*}$ ) have significantly larger dynamical ages - morphological transformations in clusters therefore proceed more quickly than in all other environments. This point is made more clearly in Fig. 3, where we plot the cumulative fraction of early-type galaxies which have already had their last merger. We find that, on average, without 


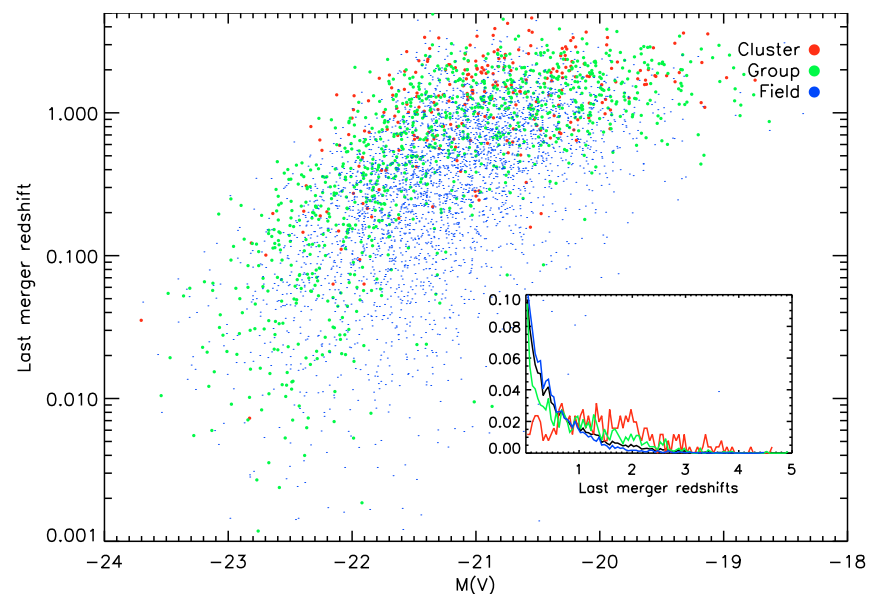

Fig. 2. Last merger redshifts of the early-types in the model, split by environment of the remnant at $z=0$. INSET: Histograms of last merger redshifts shown in the top panel, split by environment.

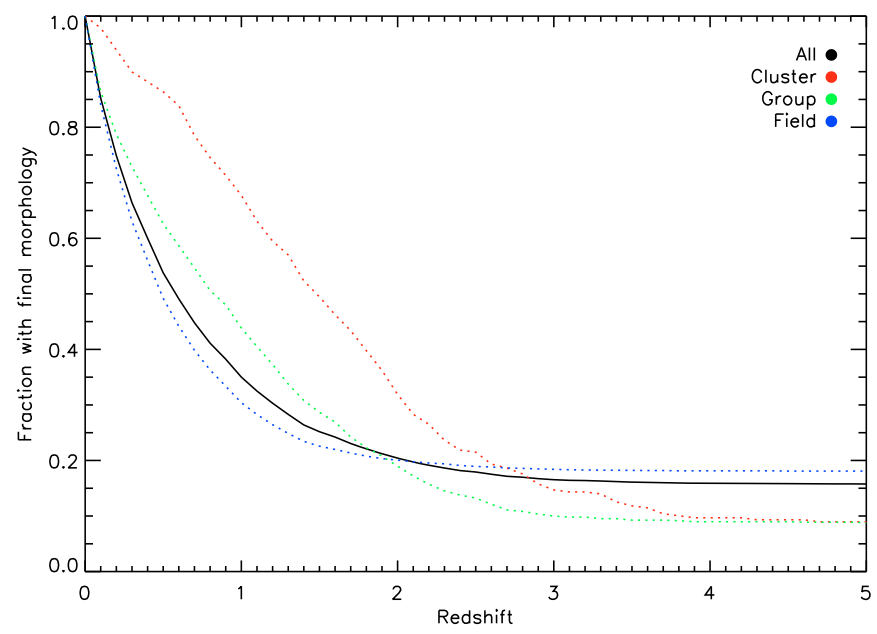

Fig. 3. Cumulative fraction of early-type galaxies which have had their last merger as a function of redshift.

reference to environment, only 35 percent of early-type galaxies are "in place" (i.e. they evolve without further interactions with other galaxies) by $z=1$ (black line). The rest are still "in pieces". In terms of morphological transformations, cluster environments are special, in that early-type morphologies are attained significantly faster in clusters (red curve), with almost 70 percent of early-type galaxies having undergone their last merger by $z=1$.

Before the last merger occurs, the morphology of the progenitors is not necessarily early-type. Figure 4 shows the morphologies of progenitors in binary mergers as a function of redshift. Non-binary mergers do happen but are rare, and only take place, in the model, at redshifts greater than 1 . In agreement with previous work (e.g. Khochfar \& Burkert 2003) we find that, in the local Universe, mergers between early-type progenitors make up less than 20 percent of the merger activity. All other mergers contain at least one spiral progenitor. Mergers involving solely spiral progenitors increasingly dominate at higher redshift and dominate the merger activity beyond $z=1$ (see also Kang et al. 2007).

Having provided a picture of the merger activity within the progenitor set, it is instructive to look at the fraction of the progenitor set which is made up of a certain morphological type as a function of redshift. Figures 5 and 6 show the number and mass

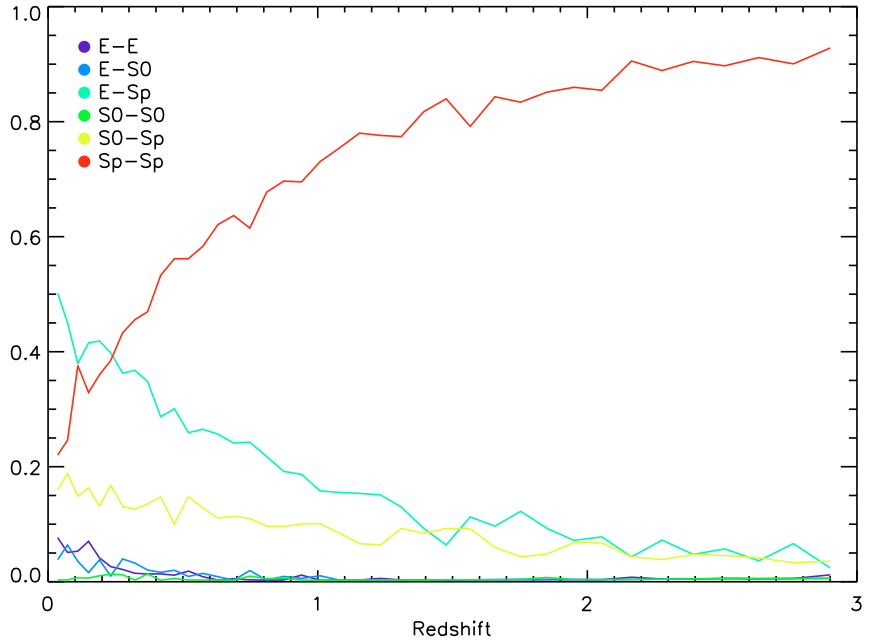

Fig. 4. Morphologies of progenitors in binary mergers as a function of redshift. Non-binary mergers do happen but are rare, and only take place, in the model, at redshifts greater than $z \sim 1.5$. Mergers at intermediate and high redshift are dominated by pairs of progenitors which contain at least one spiral progenitor.

fractions respectively of progenitors of different morphological types, in the redshift range $0<z<3$ and split by environment and luminosity of the early-type remnant at $z=0$. We find that, averaging across all environments, at $z \sim 1$, less than 50 percent of the stellar mass which ends up in early-types today is actually in early-type progenitors at this redshift. Faster morphological transformations in cluster environments means that this value is $\sim 65$ percent in clusters at $z \sim 1$. As a result, looking only at early-type galaxies at $z \sim 1$ does not take into account almost half the stellar mass in the progenitor set. In other words, the mass in the progenitor set doubles between $z=1$ and $z=0$. A similar observational result was found by Bell et al. (2004) and Faber et al. (2007), who used the optical "red sequence" as a proxy for the progenitor set of present-day early-type galaxies (see also the discussion in Sect. 6 below).

The bias does not arise simply because some progenitor mass is not taken into account, but because the age profile of the mass in progenitors of different morphological types tends to vary. We illustrate this point in Fig. 7. The top panel shows the average $N U V$-weighted ages of progenitors of different morphological types. The $N U V$ weighting, generated using the GALEX (Martin et al. 2005) $N U V$ filter (2300 angstroms), is heavily dominated by stars formed within the last $0.5 \mathrm{Gyr}$ of look-back time. At all redshifts, early-type progenitors have higher $N U V$ weighted ages, because the mass fraction contributed by recent star formation (RSF) i.e. within the last $0.5 \mathrm{Gyr}$ is smaller than for spiral progenitors. The differences between elliptical and spiral progenitors are most pronounced at low redshift. The bottom panel shows the fraction of the RSF across the progenitor set that is contained in each morphological type. This plot has to be interpreted in conjunction with the mass fractions hosted by each morphological type as a function of redshift. For example, at $z \sim 0.1$, although spiral progenitors host $\sim 40$ percent of the total RSF in the progenitor set, they only constitute $\sim 30$ percent of the mass in the progenitor set (see bottom panel of Fig. 5). Early-type progenitors (elliptical and S0 taken together) contribute $\sim 60$ percent of the RSF - but they also constitute $\sim 70$ percent of the total mass in the progenitor set. Therefore at $z \sim 0.1$ spiral progenitors host 1.5 times the amount of RSF per unit masss than their early-type counterparts. At higher redshift the 

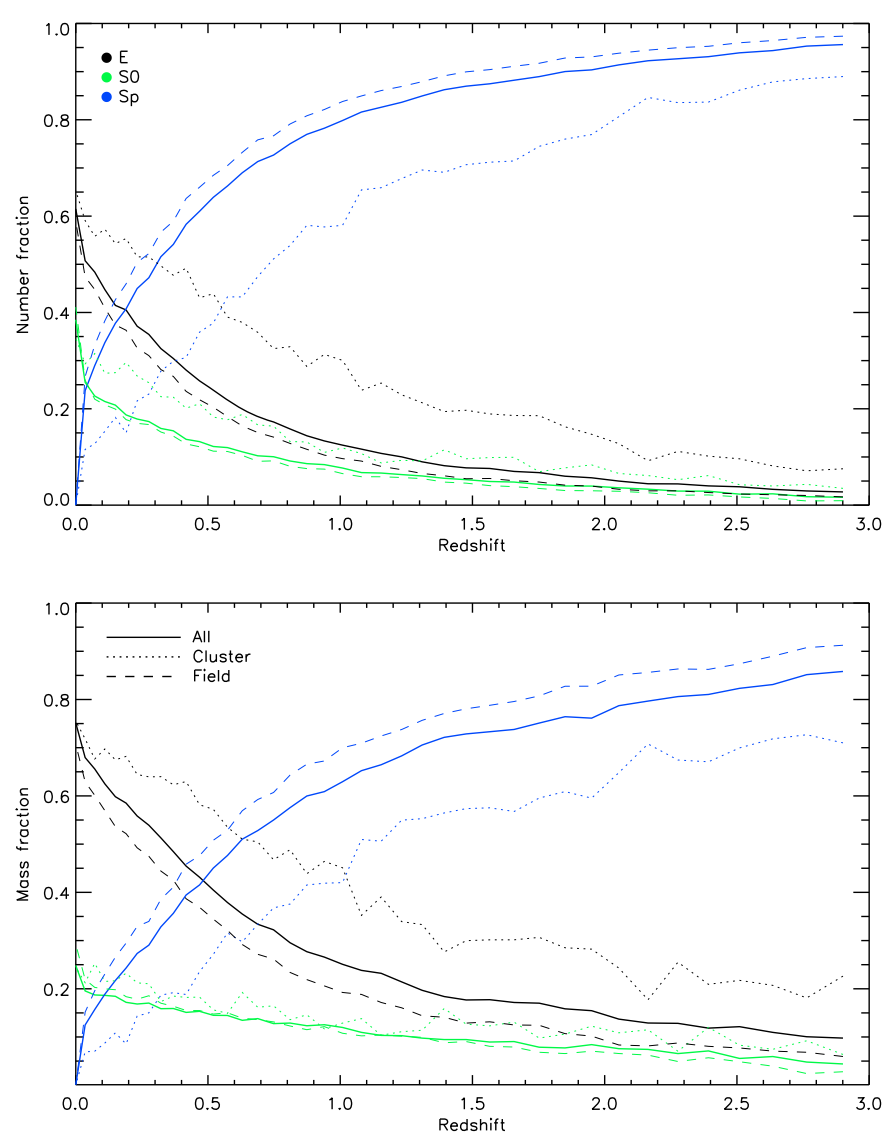

Fig. 5. Number (top) and mass (bottom) fractions contained in progenitors of different morphological types in the redshift range $0<z<3$, split by the environment of the early-type remnant.

balance of RSF contained in each morphological type moves towards spiral progenitors, partly because they are more spirals in the Universe than early-types.

Figure 7 illustrates that an increasingly larger fraction of $\mathrm{RSF}$ in the progenitor set is contained in late-type systems at increasing redshift. In the context of colour-magnitude relations (CMRs), which are often used to age-date early-type populations at all redshifts, the exclusion of spiral progenitors at high redshift biases the CMR towards redder colours and does not give a proper indication of the age of all the stellar mass that eventually constitutes present-day early-type galaxies. This is particularly true if blue filters (e.g. $U$-band or shorter wavelengths) are used in the age-dating analysis.

\section{The spiral progenitors}

One of the aims of this study is to provide a means of including spiral galaxies observed at high redshift which may be progenitors into studies of early-type galaxy evolution, and thus correct, at least partially, for progenitor bias. We therefore focus on spiral progenitors predicted by the model and compare their photometric properties to the general spiral population.

\subsection{The luminosity function of spiral progenitors}

We begin by studying the luminosity function (LF) of spiral progenitors. We are interested in studying how the luminosities of spiral progenitors compare to the general spiral population and what fraction of spirals, at a given luminosity, are
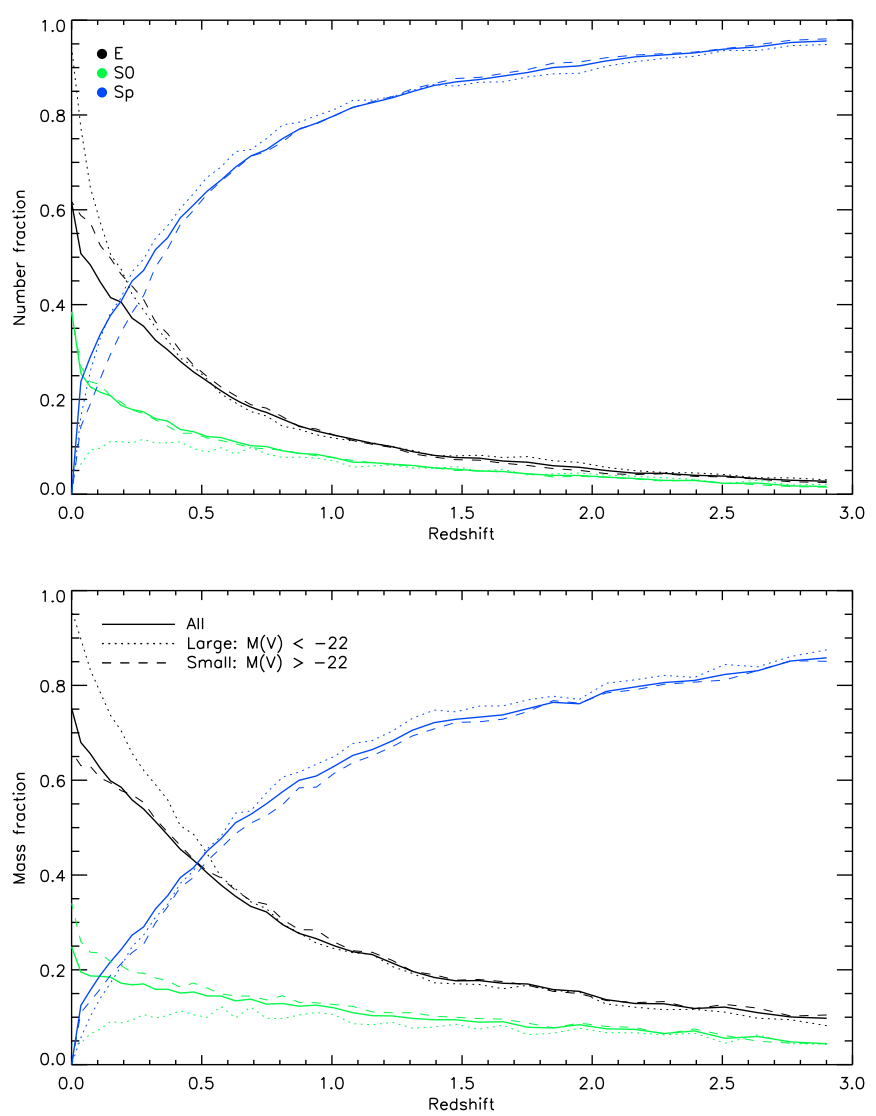

Fig. 6. Number (top) and mass (bottom) fractions contained in progenitors of different morphological types in the redshift range $0<z<3$, split by the luminosity of the early-type remnant at present-day.

early-type progenitors. In Fig. 8 we show the evolution of the $B$ band LF of spiral progenitors - we also show the spiral progenitors separated by the environment of their corresponding earlytype remnant at present-day. The left hand column illustrates the evolution of the spiral LFs - the yellow curve denotes the LF of the general spiral population (i.e. progenitors + non-progenitors) and the black curve the LF of spiral progenitors only. The LFs of spiral progenitors whose early-type remnants are, at $z=0$, in clusters, groups and the field, are shown in red, green and blue respectively. The right-hand column shows the fraction of spiral galaxies which are early-type progenitors as a function of their $B$-band luminosities. The colour coding is identical to that used for the left-hand column.

It is apparent that there is a greater preponderance of progenitors amongst larger spirals at all redshifts. For example, at low redshifts $(z<0.1), 20$ to 40 percent of spirals with $M(B)<-20.5$ are early-type progenitors. At intermediate redshifts $(0.3<z<0.52)$, these values rise to 30 and 60 percent respectively. At high redshift $(z \sim 1)$ spirals with $M(B)<-21.5$ have more than a 60 percent probability of being an early-type progenitor, while spirals with $-20<M(B)<-21.5$ have between a 30 and 40 percent chance of being early-type progenitors. The falling progenitor fractions towards lower redshift are partly due to the changing morphological mix of the Universe.

\subsection{The colour magnitude space of spiral progenitors}

While investigating the LFs of spiral progenitors is useful in indicating the probability that a spiral of a given luminosity has of being a progenitor, it is also desirable to explore the 


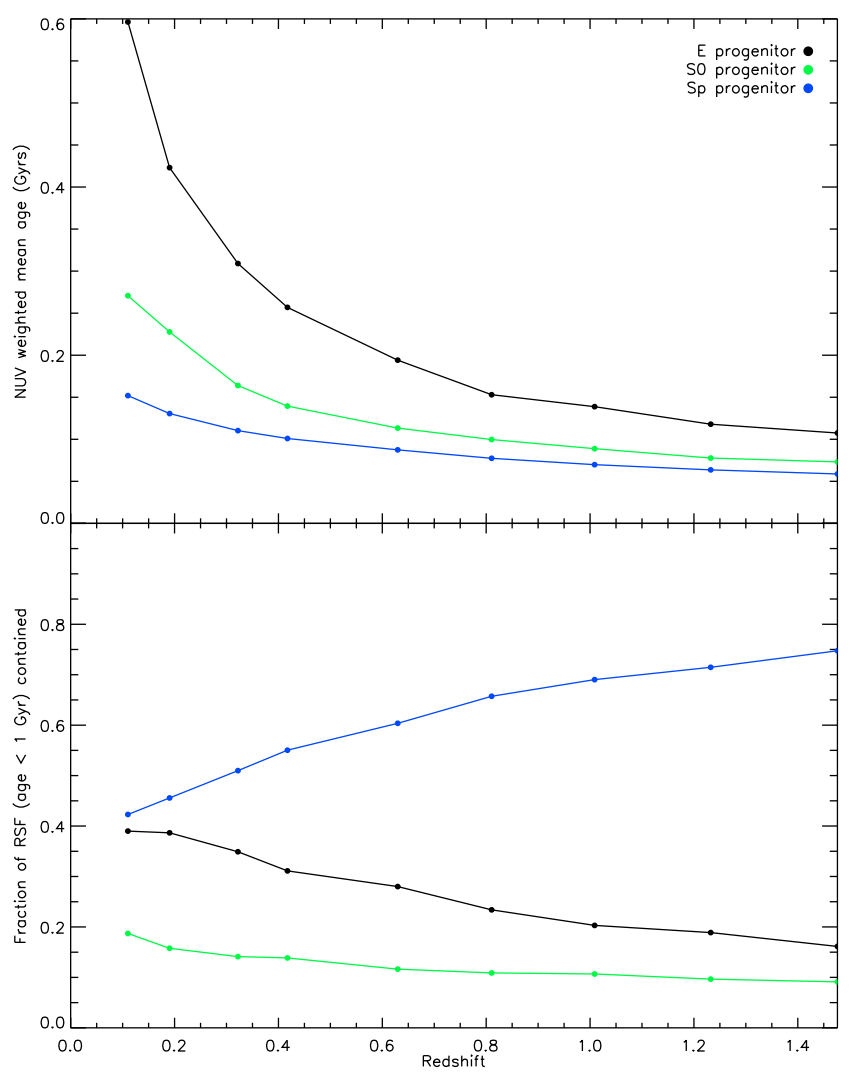

Fig. 7. Top: average $N U V$-weighted ages of progenitors of different morphological types. The $N U V$ weighting is heavily dominated by stars formed in these progenitors within the last $0.5 \mathrm{Gyr}$ of look-back time. The $N U V$ weighting was generated using the GALEX (Martin et al. 2005) NUV filter. Bottom: the mass fraction of recently formed stars (age $<1$ Gyr old) across the progenitor set which is contained in progenitors of each morphological type. Note that the bottom panel has to be interpreted in conjunction with the mass fractions hosted by each morphological type as a function of redshift. For example, at $z \sim 0.1$, although spiral progenitors host $\sim 40$ percent of the total RSF in the progenitor set, they only constitute $\sim 30$ percent of the mass in the progenitor set (see bottom panel of Fig. 5). Early-type progenitors (elliptical and S0 taken together) contribute $\sim 60$ percent of the RSF - but they also constitute $\sim 70$ percent of the total mass in the progenitor set. Therefore at $z \sim 0.1$ spiral progenitors host 1.5 times the amount of RSF per unit mass than their early-type counterparts.

colour-magnitude (CM) space of the spiral population, so that we can separate progenitor spirals better from the general population at a given luminosity.

In Fig. 9 we compare the $(B-V)$ colours of the general spiral population to the $(B-V)$ colours of spiral progenitors. The left-hand column shows the spiral $(B-V)$ CMR from $z=0$ to $z=1$. Black dots represent the spiral galaxies and red dots represent spiral progenitors. In the right hand column we show the fraction of spiral progenitors across the $(B-V)$ CMR. The fractions are indicated using the colour coding shown in the legend. Warmer colours indicate a higher progenitor fraction (red implies a progenitor fraction of 1, black represents a progenitor fraction of 0 and parts of the CM space without any galaxies are not colour-coded).

At $z \sim 0.1$, spirals with $-21.5<M(B)<-20.5$ have $\sim 30$ percent chance of being a progenitor. For larger spirals, those with red $(B-V)$ colours (i.e. $(B-V)>0.8)$ have $\sim 60$ percent chance of being a progenitor, while the corresponding probability for bluer spirals is 30 to 50 percent.
At intermediate redshift $(z \sim 0.5)$, spirals with $-21.5<$ $M(B)<-20.5$ and $(B-V)>0.6$, have $\sim 30$ percent probability of being an early-type progenitor, while blue spirals in the same luminosity range have a low progenitor probability. For larger spirals at these redshifts, the probabilities are appreciably higher - red spirals with $(B-V)>0.7$ have between a 75 and 95 percent chance of being progenitors, while 50 to 75 percent of blue spirals in this luminosity range are progenitors. The situation at high redshift $z \sim 1$ is similar to that at intermediate redshift.

\section{Progenitor evolution in clusters}

Before the advent of large scale surveys, dense regions of the Universe were often targetted for early-type galaxy studies, both at low and high redshift (e.g. Bower et al. 1992, 1998; Stanford et al. 1998; van Dokkum et al. 1998, 1999, 2000, 2001). While studies of dense regions are attractive for a variety of reasons (e.g. Ellis 2002; van Dokkum 2004), a key benefit is statistical convenience - clusters provide access to large homogeneous samples of luminous objects at all redshifts. It has been usual to "connect" results from cluster studies over large redshift ranges to determine (at least qualitatively) the chronology of galaxy evolution.

In this section, we investigate progenitors of present-day cluster early-types, which are themselves in dense regions at $z>0$. The motivation for this investigation is two fold. Firstly (and most importantly), it provides a comparison to the vast literature of "cluster" early-type studies. Secondly, this version of the GALICS model has been accurately calibrated to match the optical CMRs of early-types in dense regions from low to high redshift (Kaviraj et al. 2005), which implies that the colours of early-type progenitors (regardless of morphology) are robustly reproduced.

It is important here to clarify the definitions of "density" that we use to define both "clusters" at present-day, and "dense" regions at high redshift. As mentioned before, model "density" is assumed to be a direct function of the mass of the DM halo in which galaxies are embedded. At $z=0$, a DM halo mass of $10^{14} M_{\odot}$ represents the lower limit for a cluster-hosting halo. Observational studies of dense regions at high redshift are likely to contain an assortment of cluster-type haloes of varying occupancies. Furthermore, DM haloes themselves are evolving on average, the largest haloes at $z=1$ are likely to be roughly half their size at present day (e.g. van den Bosch 2002). To take these two points into account, we use a variable lower mass limit for "cluster-hosting" haloes at $z>0$. At a given redshift this lower limit is calculated from the average mass accretion history (van den Bosch 2002, see their Fig. 5) applied to a $10^{14} M_{\odot}$ halo.

Figure 10 shows the $(B-V)$ CMR in clusters in the redshift range $0<z<1$. Large diamonds indicate progenitor galaxies - black indicates ellipticals, green indicates SOs and blue indicates spiral galaxies. Small crosses indicate galaxies which do not contribute to the mass in present-day cluster early-types. All early-type galaxies in dense regions are, not unexpectedly, progenitors of cluster early-types at present day. The top panel in Fig. 11 shows the fraction of spiral galaxies in dense regions at high redshift (split by luminosity) which are progenitors of early-types at $z=0$. The bottom panel shows the offset in $(B-V)$, with respect to elliptical progenitors, of the S0 and spiral progenitor galaxies. The offsets are shown split by luminosity.

We find that at high redshift $(z \sim 1)$, up to 40 percent of large spirals $(-23<M(V)<-21)$ are progenitors, whereas only $\sim 10$ percent of small spirals $(M(V)>-21)$ are members of the progenitor set. Large spirals are four times more likely to be 

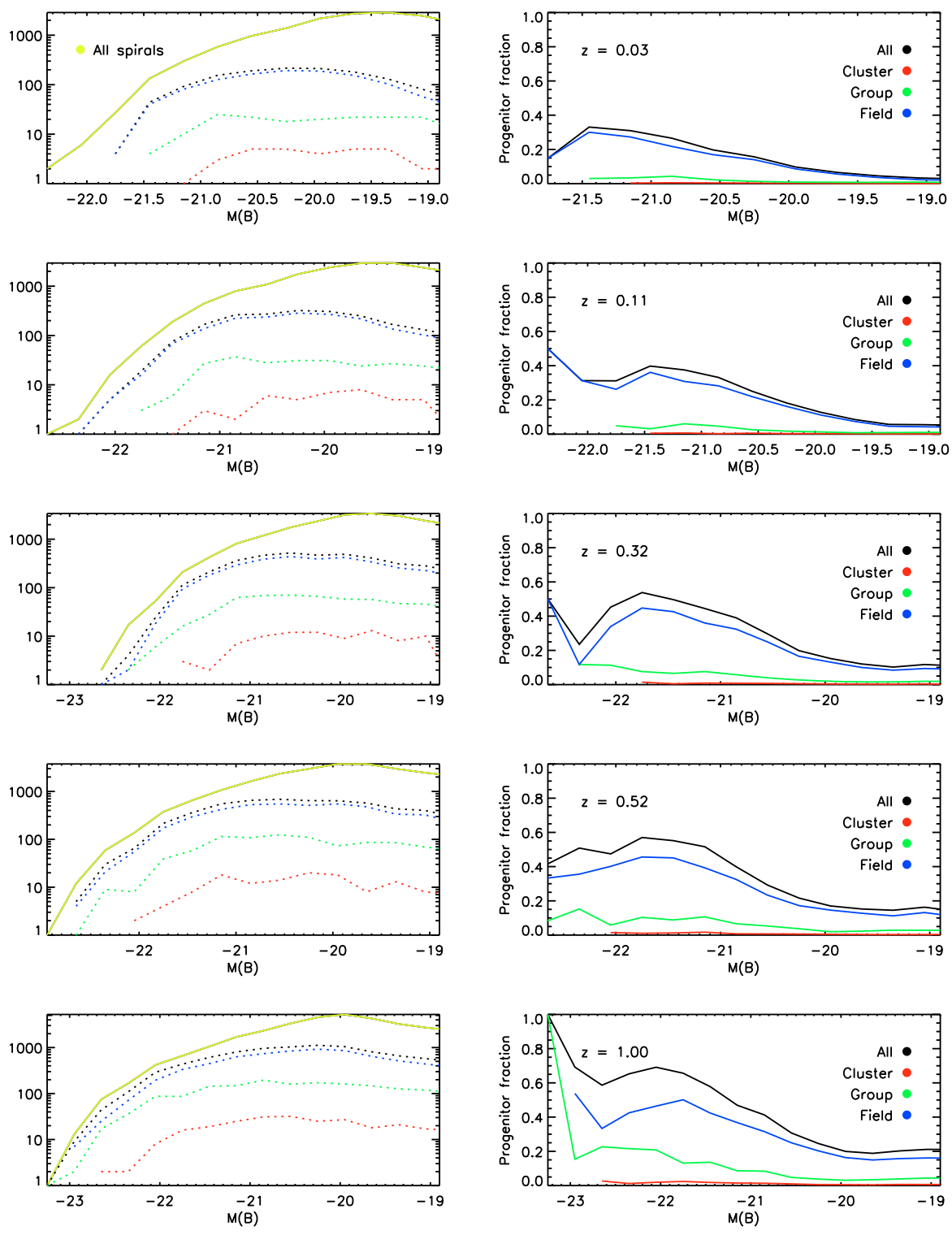

Fig. 8. Evolution of the $B$-band LF of spiral progenitors. The left hand column illustrates the evolution of the spiral LFs - the yellow curve denotes the LF of the general spiral population (i.e. progenitors + non-progenitors) and the black curve the LF of spiral progenitors only. The LFs of spiral progenitors whose early-type remnants are, at $z=0$, in clusters, groups and the field, are shown in red, green and blue respectively. The right-hand column shows the fraction of spiral galaxies which are early-type progenitors as a function of their $B$-band luminosities. The colour coding is identical to that used for the left-hand column.

progenitors than small spirals, regardless of redshift, in the redshift range $0<z<1$. Elliptical galaxies form the reddest locus in $(B-V)$. S0 galaxies show an average offset of -0.04 compared to the elliptical population, regardless of luminosity. Large spiral progenitors show an average $(B-V)$ offset of -0.05 compared to the elliptical population at high redshift, mainly because the scatter in the elliptical colours also tends to be large at high redshift. At low redshift the offset is more pronounced - large spiral progenitors are up to 0.1 mag bluer in $(B-V)$ than the elliptical population.

\section{The "red sequence" as a proxy for the progenitor set}

Early-type galaxies in clusters tend to preferentially populate the reddest parts of the colour-magnitude $(\mathrm{CM})$ space. In this section we investigate whether the sample of galaxies (without reference to morphology), within the "red sequence" can be used as a proxy for the progenitor set. We define the red sequence as the galaxy population which occupies the part of the CM space which is dominated by early-type galaxies. In Figs. 12 and 13 the CM space dominated by early-type galaxies is shown in grey - this region is determined by a progressive one-sigma fit to the colours of the early-type population. It therefore contains, on average, 68 percent of the early-type population within it. Large diamonds indicate galaxies which are part of the progenitor set. Galaxies which are not part of the progenitor set are shown using small dots. Galaxies in the red sequence are circled. It is apparent that the red sequence misses blue progenitor galaxies, both early-type and late-type. Figure 12 shows the evolution of the red sequence population from low to intermediate redshift and Fig. 13 the corresponding evolution from intermediate to high redshift.

In Fig. 14 we compare galaxies in the actual progenitor set to those in the red sequence. The top panel shows the number ratio between the progenitor set population and the red sequence population, split by redshift and luminosity, while the bottom panel shows the mass ratio between the progenitor set population and the red sequence population split by redshift and luminosity. It is apparent that large galaxies $(-23<M(V)<-21)$ in the red sequence trace the progenitor set well in terms of number and 


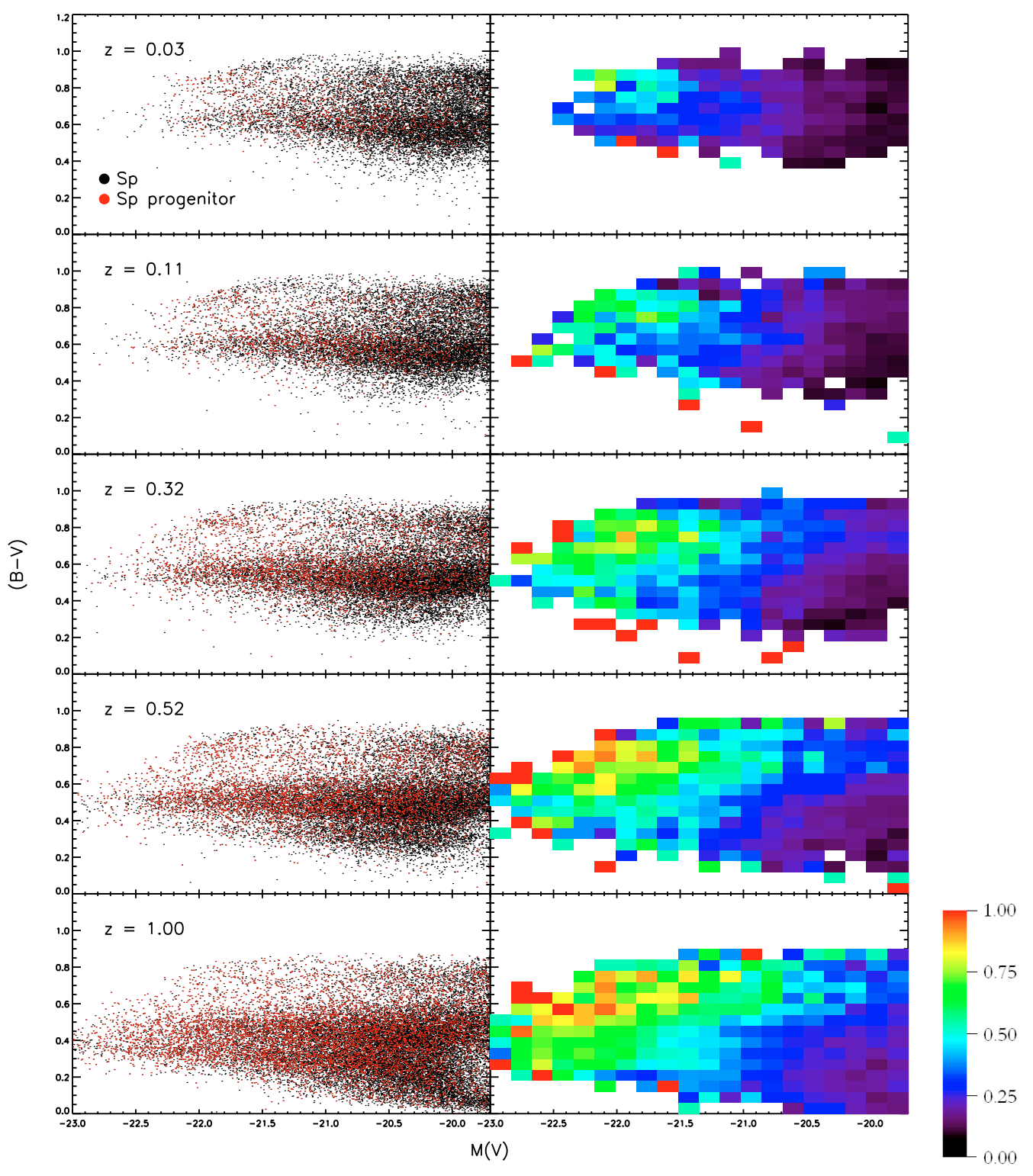

Fig. 9. $(B-V)$ colours of the general population compared to the $(B-V)$ colours of those spirals which are progenitors of present-day earlytype galaxies. Black dots represent spiral galaxies and red dots represent spiral progenitors. The left-hand column shows the spiral $(B-V)$ CMR from $z=0$ to $z=1$. In the right hand column we show the corresponding (binned) fraction of spiral progenitors across the $(B-V)$ CM space. The fraction values are indicated using the colour coding shown in the legend. Warmer colours indicate a higher progenitor fraction (red implies a progenitor fraction of 1, black represents a progenitor fraction of 0 and parts of the CM space without any galaxies are not colourcoded). mass but that the relationship breaks down as we move towards the lower end of the luminosity function $(M(V)>-21)$. The number and mass fractions remain stable, as a function of the luminosity slices shown in Fig. 14, within the redshift range explored in this study $(0<z<1)$.

Luminosity evolution studies which use the red sequence as a proxy for the early-type population can therefore achieve accurate results only for the massive end of the luminosity function. Note that the results of two such recent studies, Bell et al. (2004) and Faber et al. (2007), are accurate since their analysis of the luminosity evolution of red galaxies is indeed restricted to massive galaxies on the red sequence (e.g. $M(V) \leq-21$ in Bell et al. 2004). However, it should be noted that the red sequence is less reliable as a proxy for the progenitor set further down the luminosity function. In addition, the red sequence, almost by definition, misses contributions due to early-types which lie blueward of it. This is increasingly true at higher redshift - hence conclusions based on the colours of the red sequence should be applied carefully to early-type evolution as deeper or higher redshift data becomes available in the future.

\section{Summary and discussion}

We have presented a comprehensive theoretical study, using a realistic semi-analytical model in the framework of the standard LCDM paradigm, of the photometric properties of the progenitors of present-day early-type galaxies. We have mapped, in detail, the properties of these progenitors, regardless of their morphology, in the redshift range $0<z<1$ (where the bulk of current large-scale surveys are focussed), as a function of the luminosity and environment of the early-type remnant at present day. We have also developed probabilistic prescriptions which provide a means of including spiral (i.e. non early-type) progenitors at intermediate and high redshifts, based on their luminosity and optical $(B V K)$ colours. The prescriptions developed here can potentially be used to address, from the perspective of the standard modelss, the issue of "progenitor bias", whereby the exclusion of late-type progenitors in observational studies can lead to inaccurate conclusions regarding the evolution of the earlytype population over cosmic time. While simple prescriptions designed to alleviate progenitor bias do exist and have been used 


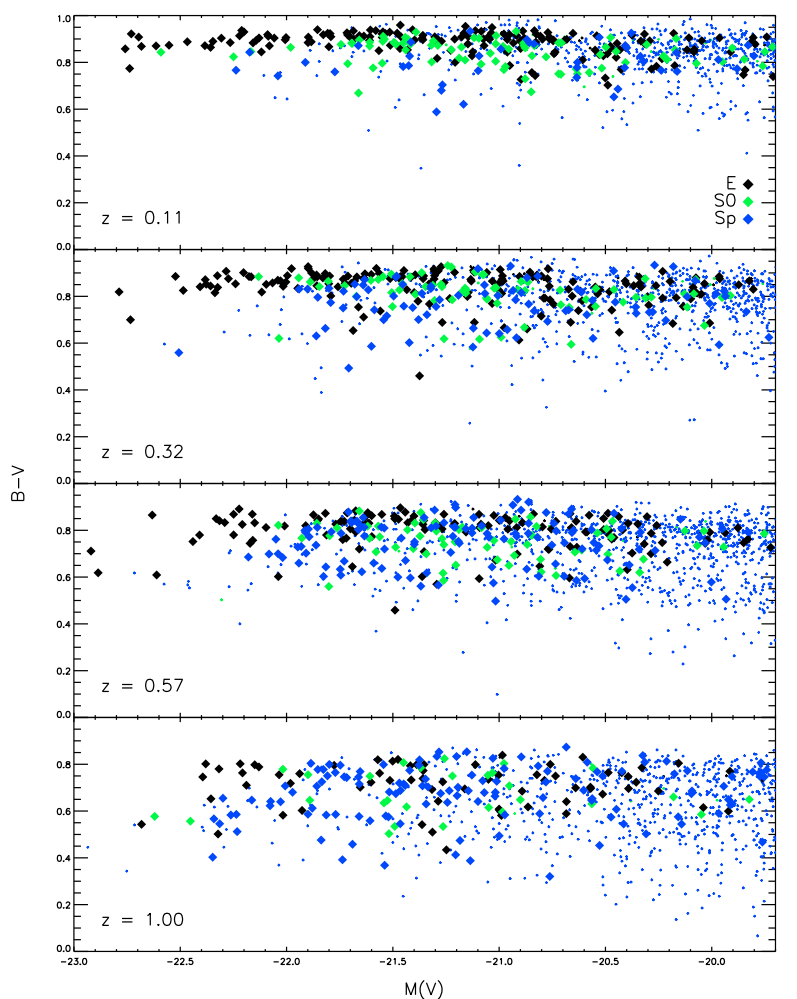

Fig. 10. The $(B-V)$ CMR in clusters in the redshift range $0<z<1$. Large diamonds indicate the progenitors of present-day cluster earlytype galaxies. Small crosses indicate galaxies which do not contribute to the mass in present-day cluster early-types. Note that all elliptical and S0 galaxies in dense regions are, not unexpectedly, progenitors of present-day cluster early-types.

in some observational studies, they do not reflect the mass assembly and morphological transformations inherent to the standard model.

Against the backdrop of the impending or recent release of data from large-scale surveys at high redshift, the results of this study are hopefully timely, providing a picture of how the mass in present-day early-types, across a range of luminosities and environments, have assembled and exploring the extent to which spiral progenitors play a role in this process. Our main conclusions can be summarised as follows:

- Larger early-types in all environments are assembled later than their less massive counterparts. However, their stellar populations are generally older. This result is consistent with previous studies of early-type galaxies in the semi-analytical framework (e.g. Kauffmann et al. 1996; Baugh et al. 1996; Kaviraj et al. 2005; De Lucia et al. 2006).

- On average, without reference to environment, only 35 percent of early-type galaxies are in place by $z=1$ and evolve without further interactions with other galaxies thereafter. Morphological transformations are significantly faster in cluster environments (where the vast majority of early-type studies have been based before the advent of large-scale surveys). In clusters almost 70 percent of early-types are in place by $z=1$. In other words, the probability of a "major merger", which creates an early-type remnant is low after $z=1$ in cluster type environments.

- Averaging across all environments, at $z \sim 1$, less than 50 percent of the stellar mass which ends up in early-types

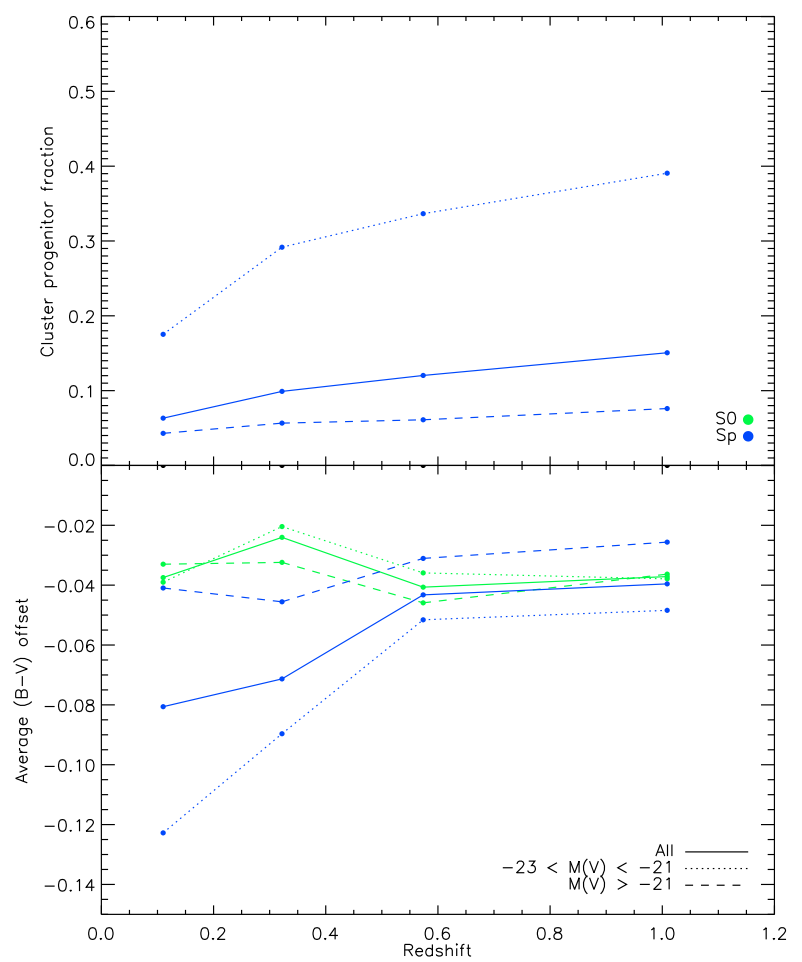

Fig. 11. Top panel: the fraction of spiral galaxies in dense regions (split by luminosity) which are progenitors of cluster early-types at $z=0$. Note that all elliptical and S0 galaxies in dense regions are, not unexpectedly, progenitors of present-day cluster early-types. Bottom panel: the offset in average $(B-V)$ colour, with respect to elliptical progenitors, of S0 and spiral progenitors in dense regions. The offsets are shown split by luminosity.

today is actually in early-type progenitors at this redshift. This value is around 65 percent in clusters owing to faster morphological transformation in this environment. In other words, looking only at early-type progenitors does not take into account almost half the mass in the progenitor set the progenitor set doubles in mass in the redshift range $0<z<1$.

- Progenitor bias does not arise simply because (late-type) progenitor mass is missed, but also because the age profile of mass in progenitors of different morphological types tend to vary. Spiral progenitors are typically "bluer" (at a given redshift) because they host more recently formed stars than early-type progenitors. Hence, age-dating the progenitor set using an "early-type only" CMR, i.e. after excluding the spiral progenitors, biases the CMR towards redder colours and overestimates the average age of the progenitor set.

- One of the aims of this study is to provide a means of including spiral progenitors into studies of early-type evolution, and thus correct, at least partially, for progenitor bias. We have therefore focussed on spiral progenitors in the model and compared their properties, in detail, to the general spiral population.

- There is a greater preponderance of progenitors amongst larger spirals at all redshifts. At low redshifts $(z<0.1), 20$ to 40 percent of spirals with $M(B)<-20.5$ are early-type progenitors. At intermediate redshifts $0.3<z<0.5$, these values rise to 30 and 60 percent respectively. At high redshift $(z \sim 1)$ spirals with $M(B)<-21.5$ have more than 


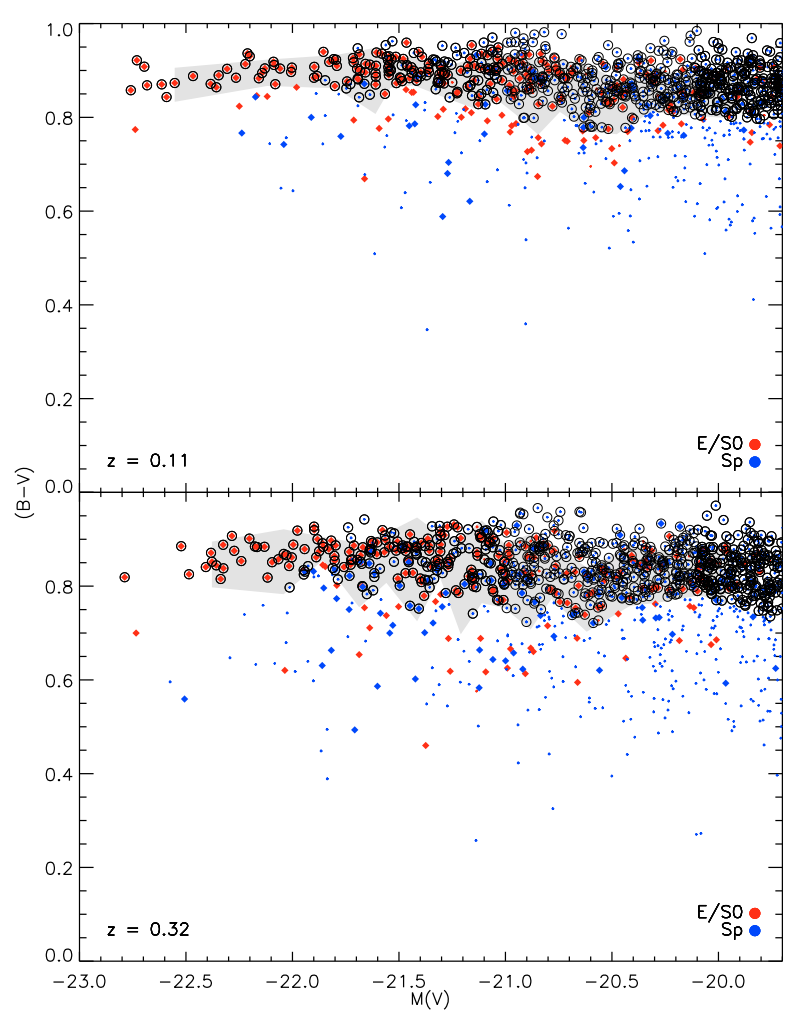

Fig. 12. The composition of the "red sequence", defined as the galaxy population which occupies the part of the $\mathrm{CM}$ space dominated by early-type galaxies (shown in grey), compared with the progenitor set in cluster populations. The diamonds indicate galaxies which are part of the progenitor set. Galaxies which are not part of the progenitor set are shown using small crosses. Galaxies in the red sequence are circled. The "red sequence" misses blue galaxies, both early-type and late-type. The top panel shows the "red sequence" at $z=0.11$, while the bottom panel shows the "red sequence" at $z=0.32$.

a 60 percent probability of being an early-type progenitor while spirals with $-20<M(B)<-21.5$ have between a 30 and 40 percent chance of being early-type progenitors. The falling progenitor fractions towards lower redshift are partly due to the changing morphological mix of the Universe.

- The colour-magnitude space of the spiral population provides a slightly better route to identifying spiral progenitors. At $z \sim 0.1$, spirals with $-21.5<M(B)<-20.5$ have $\sim 30$ percent chance of being a progenitor. For larger spirals, those with red $(B-V)$ colours, i.e. $(B-V)>0.8$, have $\sim 60$ percent chance of being a progenitor, while the corresponding probability for bluer spirals is 30 to 50 percent.

At intermediate redshift $(z \sim 0.5)$, spirals with $-21.5<$ $M(B)<-20.5$ and $(B-V)>0.6$, have $\sim 30$ percent probability of being an early-type progenitor, while blue spirals in the same luminosity range have a low progenitor probability. For larger spirals at these redshifts, the probabilities are appreciably higher - red spirals with $(B-V)>0.7$ have between a 75 and 95 percent chance of being progenitors, while 50 to 75 percent of blue spirals in this luminosity range are progenitors. The situation at high redshift $z \sim 1$ is similar to that at intermediate redshift. The trends in the $(V-K)$ colour are similar to those in $(B-V)$.

- We suggest one example of how the probabilites derived in Sect. 4 could be used to "include" spiral progenitors at high

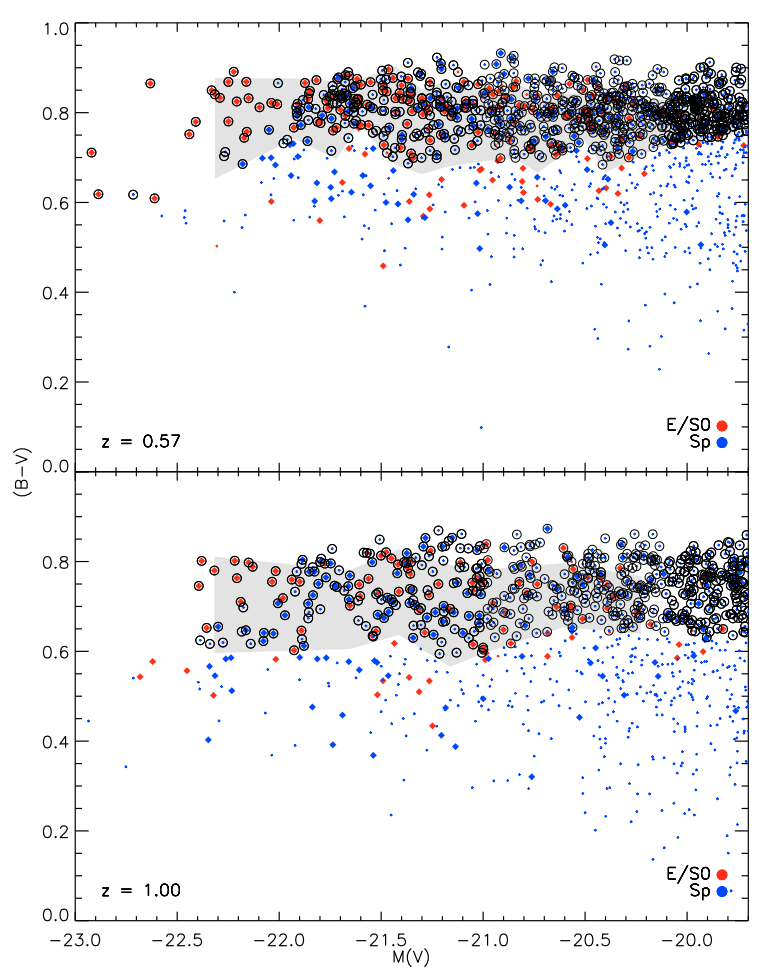

Fig. 13. The composition of the "red sequence", defined as the galaxy population which occupies the part of the CM space dominated by early-type galaxies (shown in grey), compared with the progenitor set in cluster populations. The diamonds indicate galaxies which are part of the progenitor set. Galaxies which are not part of the progenitor set are shown using small crosses. Galaxies in the red sequence are circled. The "red sequence" misses blue galaxies, both early-type and late-type. This plot shows the evolution of the "red sequence" from intermediate to high redshift. The top panel shows the "red sequence" at $z=0.57$, while the bottom panel shows the "red sequence" at $z=1$.

redshifts $(z>0.5)$. Modern optical surveys (e.g. MUSYC) can be used to trace the rest-frame $U V$ photometry of galaxies at high redshifts. Given its sensitivity to young stars, the rest-frame $U V$ is an excellent photometric tracer of recent star formation, i.e. the mass fraction of stars formed within the last Gyr. Clearly, if one had access to the rest-frame $U V$ over a range of redshifts, one could progressively trace the build-up of stellar mass in a particular class of galaxy over time. Thus, the stellar mass build-up in the early-type progenitor set can be approximated by the sum of the recent star formation in early-type galaxies within a given redshift range plus the recent star formation in spiral galaxies weighted by their relevant probabilities of being progenitors derived in Sect. 4.

- We note that the analysis presented in this paper strongly implies that model and observed early-type populations should only be compared once they have been split by environment, since the assembly history of early-type galaxies is a function of environment. For example, the combined model population (at any redshift) should not be compared to an observed population which may be drawn predominantly from rich groups and clusters and vice-versa.

- Finally we have explored the correspondence between the progenitor set and the "red sequence", defined as the part of the colour-magnitude parameter space which is dominated 

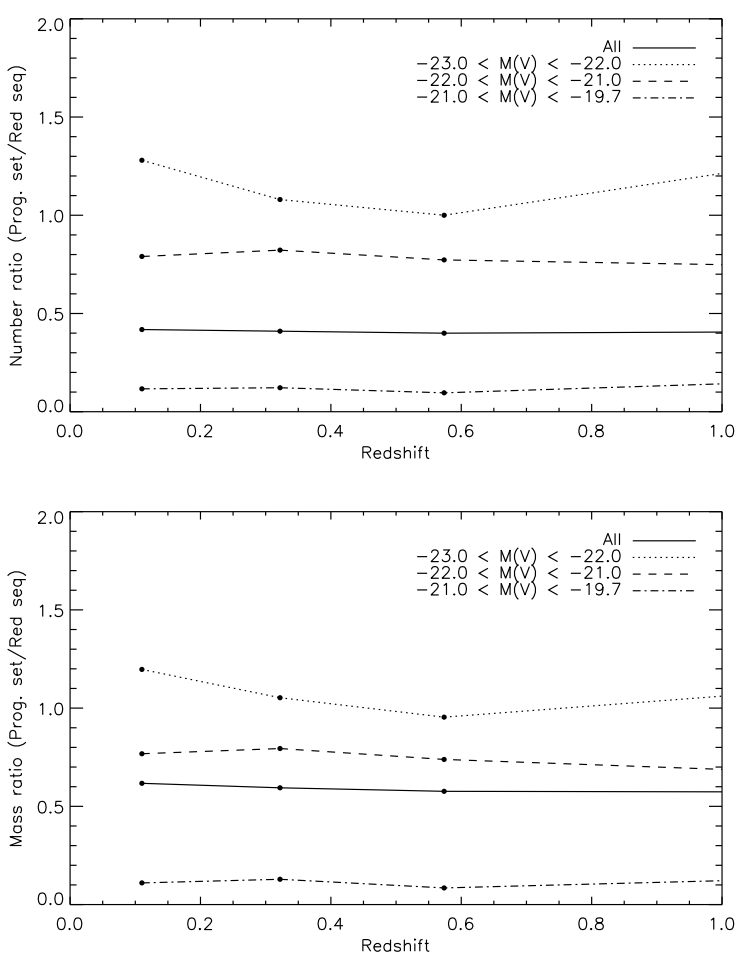

Fig. 14. Comparison between galaxies in the actual progenitor set to those in the red sequence. Top: number ratio between the progenitor set population and the red sequence population split by redshift and luminosity. Bottom: mass ratio between the progenitor set population and the red sequence population split by redshift and luminosity. It is apparent that large galaxies in the red sequence trace the progenitor set well in terms of number and mass but that the relationship breaks down rather rapidly as we move towards the lower end of the luminosity function.

by early-type galaxies. We find that galaxies, both late and early-type, that fall in this parameter space do not necessarily trace the progenitor set well. Large galaxies $(-23<$ $M(V)<-21)$ in the red sequence correspond to the progenitor set reasonably well in terms of number and mass but the relationship breaks down as we go towards the lower end of the luminosity function $(M(V)>-21)$. Hence, luminosity evolution studies which use the red sequence as a proxy for the early-type population, therefore achieve accurate results only for the upper end of the luminosity function. In addition, the red sequence, almost by definition, misses contributions due to early-types which lie blueward of it - hence conclusions based on the colours of the red sequence should not generally be applied to early-type evolution, especially at high redshift.

Acknowledgements. We are grateful to the anonymous referee for a thoughtful review which added clarity to the original manuscript. S.K. would like to thank Jérémy Blaziot for his generous help with implementing GALICS and acknowledges a Research Fellowship from the Royal Commission for the Exhibition of 1851, a Senior Research Fellowship from Worcester College, Oxford and support from the BIPAC institute at Oxford. Part of this work was supported by a Leverhulme Early-Career Fellowship (S.K., till Oct 2008). SKY was supported by the Korea Research Foundation Grant funded by the Korean government (KRF-C00156). We thank Roger Davies, Rachel Somerville, Richard Bower, Daniel Thomas, Claudia Maraston, Christian Wolf, Kevin Schawinski and Sadegh Khochfar for constructive comments on the manuscript and the work that led up to it.

\section{References}

Adelman-McCarthy, J. K., \& SDSS collaboration 2006, ApJS, 162, 38 Barrientos, L. F., \& Lilly, S. J. 2003, ApJ, 596, 129

Baugh, C. M., Cole, S., \& Frenk, C. S. 1996, MNRAS, 283, 1361 Bell, E. F., \& GEMS collaboration 2004, ApJ, 608, 752 Bertschinger, E. 1985, ApJS, 58, 1

Blakeslee, J. P., Franx, M., \& Postman, M. 2003, ApJ, 596, L143 Bower, R. G., Lucey, J. R., \& Ellis, R. 1992, MNRAS, 254, 589 Bower, R. G., Kodama, T., \& Terlevich, A. 1998, MNRAS, 299, 1193 Bower, R. G., Benson, A. J., Malbon, R., et al. 2006, MNRAS, 370, 645 Bruzual, G., \& Charlot, S. 2003, MNRAS, 344, 1000

Buta, R., Mitra, S., de Vaucouleurs, G., \& Corwin, H. G. 1994, AJ, 107, 118 Chiosi, C., \& Carraro, G. 2002, MNRAS, 335, 335

Cole, S., Lacey, C. G., Baugh, C. M., \& Frenk, C. S. 2000, MNRAS, 319, 168

Combes, F., Mamon, G., \& Charmandaris, V. 2000, PASP, 112, 423

Cross, N., \& 2dF collaboration 2001, MNRAS, 324, 825

De Lucia, G., Springel, V., White, S. D. M., Croton, D., \& Kauffmann, G. 2006, MNRAS, 366, 499

Desert, F.-X., Boulanger, F., \& Puget, J. L. 1990, A\&A, 237, 215

Devriendt, J. E. G., Guiderdoni, B., \& Sadat, R. 1999, A\&A, 350, 381

Eke, V. R., Cole, S., \& Frenk, C. S. 1996, MNRAS, 282, 263

Ellis, R. S. 2002, in Tracing Cosmic Evolution with Galaxy Clusters, ASP Conf. Ser. 268, 311

Faber, S. M., \& DEEP2 collaboration 2007, ApJ, 665, 265

Ferreras, I., Saha, P., \& Williams, L. L. R. 2005, ApJ, 623, L5

Franx, M., \& van Dokkum, P. G. 1996, in New Light on Galaxy Evolution, ed. R. Bender, \& R. L. Davies, IAU Symp., 171, 233

Gawiser, E., \& MUSYC Team 2006, ApJS, 162, 1

Gladders, M. D., Lopez-Cruz, O., Yee, H. K. C., \& Kodama, T. 1998, ApJ, 501, 571

Guiderdoni, B., \& Rocca-Volmerange, B. 1987, A\&A, 186, 1

Hatton, S., Devriendt, J. E. G., Ninin, S., et al. 2003, MNRAS, 343, 75

Holden, B. P., van der Wel, A., Franx, M., et al. 2005, ApJ, 620, L83

Kang, X., van den Bosch, F. C., \& Pasquali, A. 2007, MNRAS, 381, 389

Kauffmann, G., \& Charlot, S. 1998, MNRAS, 297, L23

Kauffmann, G., White, S. D. M., \& Guiderdoni, B. 1993, MNRAS, 264, 201

Kauffmann, G., Charlot, S., \& White, S. D. M. 1996, MNRAS, 283, L117

Kauffmann, G., Colberg, J. M., Diaferio, A., \& White, S. D. M. 1999, MNRAS, 303,188

Kaviraj, S., \& GALEX Science Team 2007, ApJS, 173, 619

Kaviraj, S., Devriendt, J. E. G., Ferreras, I., \& Yi, S. K. 2005, MNRAS, 360, 60

Kennicutt, Jr., R. C. 1983, ApJ, 272, 54

Kennicutt, Jr., R. C. 1998, ApJ, 498, 541

Khochfar, S., \& Burkert, A. 2003, ApJ, 597, L117

Larson, R. B. 1975, MNRAS, 173, 671

Lintott, C. J., Schawinski, K., Slosar, A., et al. 2008, MNRAS, 389, 1179

Loveday, J., Peterson, B. A., Maddox, S. J., \& Efstathiou, G. 1996, ApJS, 107, 201

Makino, J., \& Hut, P. 1997, ApJ, 481, 83

Maraston, C. 2005, MNRAS, 362, 799

Martin, C. L., Kobulnicky, H. A., \& Heckman, T. M. 2002, ApJ, 574, 663

Martin, D. C., \& GALEX Team 2005, ApJ, 619, L1

Rix, H.-W., \& GEMS Team 2004, ApJS, 152, 163

Salpeter, E. E. 1955, ApJ, 121, 161

Shioya, Y., \& Bekki, K. 1998, ApJ, 504, 42

Silk, J. 2001, MNRAS, 324, 313

Silk, J. 2003, MNRAS, 343, 249

Simien, F., \& de Vaucouleurs, G. 1986, ApJ, 302, 564

Somerville, R. S., \& Primack, J. R. 1999, MNRAS, 310, 1087

Stanford, S. A., Eisenhardt, P. R., \& Dickinson, M. 1998, ApJ, 492, 461

Tytler, D., Fan, X.-M., \& Burles, S. 1996, Nature, 381, 207

van de Ven, G., van Dokkum, P. G., \& Franx, M. 2003, MNRAS, 344, 924

van den Bosch, F. C. 1998, ApJ, 507, 601

van den Bosch, F. C. 2002, MNRAS, 331, 98

van Dokkum, P. 2004, in Wide-Field Imaging From Space

van Dokkum, P. G., \& Franx, M. 2001, ApJ, 553, 90

van Dokkum, P. G., \& van der Marel, R. P. 2007, ApJ, 655, 30

van Dokkum, P. G., Franx, M., Kelson, D. D., \& Illingworth, G. D. 1998, ApJ, 504, L17

van Dokkum, P. G., Franx, M., Fabricant, D., Kelson, D. D., \& Illingworth, G. D. 1999, ApJ, 520, L95

van Dokkum, P. G., Franx, M., Fabricant, D., Illingworth, G. D., \& Kelson, D. D. 2000, ApJ, 541, 95

van Dokkum, P. G., Stanford, S. A., Holden, B. P., et al. 2001, ApJ, 552, L101

Walker, I. R., Mihos, J. C., \& Hernquist, L. 1996, ApJ, 460, 121

Wolf, C., Meisenheimer, K., Kleinheinrich, M., et al. 2004, A\&A, 421, 913

Yi, S. K. 2003, ApJ, 582, 202 